\title{
Multislot Antenna with a Screening Backplane for UWB WBAN Applications
}

\author{
Yun-fei Wei and Christophe Roblin \\ COMELEC Department, Télécom ParisTech, 46, rue Barrault, 75013 Paris, France \\ Correspondence should be addressed to Christophe Roblin, christophe.roblin@telecom-paristech.fr
}

Received 15 May 2012; Revised 8 August 2012; Accepted 23 August 2012

Academic Editor: Z. N. Chen

Copyright (๑) 2012 Y.-f. Wei and C. Roblin. This is an open access article distributed under the Creative Commons Attribution License, which permits unrestricted use, distribution, and reproduction in any medium, provided the original work is properly cited.

\begin{abstract}
A novel multislot antenna with a conducting backplane reflector (MSA-BP) designed for ultrawideband wireless body area networks (WBANs) applications is presented. The objective is to achieve a desensitization of the antenna behavior regarding the proximity effects of the human body, thanks to the field screening operated by the backplane. Partly because of the latter, the antenna is fed with a tapered CPW. The influence of antenna parameters and human body proximity on the radiation characteristics of the MSA-BP is analyzed. Simulated results of the antenna close to a three-layer arm phantom are presented. Impedance and radiation measurements of the isolated antenna as well as propagation characteristics along the human body demonstrating the desensitization effect are also presented. The characteristics of the MSA-BP, in terms of the on-body total radiation efficiency, are significantly improved compared to any (quasi-) omnidirectional counterpart.
\end{abstract}

\section{Introduction}

Wireless body area networks (WBANs) are systems of wireless communication between wearable or implantable electronic devices (and possibly an off-body access point). Such systems are promising candidates for many applications as diverse as medical sensor systems, sport monitoring, personal entertainment, and military domain (soldier equipment in particular) $[1,2]$. In recent years the ultra-wideband (UWB) technology, thanks to its low radiated power levels (as well as localization capabilities and potential high-data rates), has received great interest in the wireless body area networks $[3,4]$. Antennas play an important role in communication systems, notably UWB, and more specifically in impulse radio-based systems for which the antenna characteristics (in particular in the time domain $[5,6]$ ) interact with those of the RF front-end and propagation channel $[7,8]$. In addition, beyond usual design criterions which hold as well for UWB antennas (impedance matching, radiation performance, through the realized gain and efficiency [9]), specific requirements for integration in small devicesthe most common case in the WBAN context-impose size constraints and easy integration with circuits. Several
UWB antennas have been designed for WBAN applications $[10,11]$. For example in [10], an interesting low profile monopole-like planar antenna is designed in order to excite a TM mode - with the $\mathbf{H}$ field tangent to the body - known to be dominant along the body $[1,12]$. However, although very compact (with a height of $10 \mathrm{~mm}$ ), its main drawback is that it is "protruding," and consequently restricted to an integration in rather thick devices. In addition, extra losses caused by power absorption of the human body, as well as diffraction and reflection effects due to its high permittivity, degrades the antenna overall total efficiency and radiation characteristics [13-15]. These physical phenomena have of course a significant negative impact on the radio link performance. In particular the characteristics of the WBAN channel, which are very specific, are intimately related to antennas performance $[16,17]$. All this calls for a dedicated design of antennas for WBAN. However, this approach has been adopted only recently in the literature. Previous works have been limited to using antennas previously designed (notably UWB) and to characterize their performance in the WBAN context.

Antennas can be classified as electric (dipoles, horns...) or magnetic (loops, slots...) [18], for the first the stored 
energy in the reactive zone is dominantly electric, whereas it is dominantly magnetic for the last. Due to its "dielectric nature," the proximity of a human subject can strongly affect the input impedance of electric antennas, whereas it is less true for magnetic ones [1,14-17]. As a consequence, loop and slot antennas are interesting candidates for WBAN applications.

However, a challenge for the design of WBANs antennas remains their potential sensitivity to the proximity of the human subject. For narrowband (NB) antennas, input matching is of major concern, as detuning is the main effect (the matching frequency decreasing as the antenna/body spacing is reduced). Indeed, irrespective of other effects such as losses or increased directivity, the strong influence of the body in the antenna reactive zone $[13,16,17,19,20]$ can lead to a collapse of the energy transfer because of an abrupt increase of the reflection coefficient. Consequently, for NB antennas, resorting to desensitization techniques with respect to the body proximity is mandatory. Several strategies are possible, the most common consists in broadening the bandwidth and other approaches are based on the screening of the field. For the last, several techniques have been proposed: adding a cavity [21] or a shielding plane behind the antenna or using absorbing materials, which could increase the antenna thickness and may complicate integration with WBAN devices. However, this is not necessarily the case, for example, recently, a technique using ferrite sheets glued on one side of planar antennas, that is, introduced between the antenna and the body, has been applied successfully [22] (without significantly increasing the thickness). The price is a drop of the antenna efficiency (due to the ferrite losses), but this is largely compensated by the reduction of losses due to the body and increased robustness against detuning. In the UWB context, the matching requirements are less stringent. Additional losses due to the body tend to lower globally the $\left|S_{11}\right|$, whereas the high permittivity of the body - acting as a sort of additional substrate — tends to shift down the pass band, the combined effects resulting in a decrease of the lower cutoff frequency and a shift of the upper cutoff frequency in one or another direction. Moreover, the matching can be locally improved or degraded, but generally not dramatically [17].

An alternative approach to reduce the backward radiation is to use a reflector element $[23,24]$. These antennas are efficient regarding the reduction of the backward radiation, but are still relatively thick $(7.58 \mathrm{~mm}$ and $8.76 \mathrm{~mm}$ resp.), although remaining compatible with integration in WBAN devices.

To our knowledge, there is only a very few UWB slot antennas (notably in the $3-6 \mathrm{GHz}$ range) which have been designed with a backplane reflector In addition their purpose was mainly to achieve unidirectionality and have not been applied to the WBAN context $[25,26]$. The antenna with a backplane in [25] operates over the highest UWB subband $(7.25-10.25 \mathrm{GHz})$, whereas the antenna with a planar reflector in [26] operates over $0.8-2.3 \mathrm{GHz}$ for a through-the-wall radar application.

In this paper, a novel multiple slot antenna (MSA-BP), operating from 3.38 to $6.07 \mathrm{GHz}$, is proposed for UWB
WBAN applications. A backplane (metallic reflector) [25, 26], with the purpose of achieving a significant reduction of the backward radiation is introduced, producing a screening effect between the radiation and the body. By means of a commercial full-wave electromagnetic (EM) numerical solver (CST Microwave Studio), using the FIT (Finite Integral Technique), the influence of the backplane and the other design parameters on the characteristics of the proposed antenna is investigated. Further numerical analyses are led with an arm phantom, in order to investigate the onbody propagation performance.

The paper is organized as follows: in Section 2, the antenna design is detailed with a parametric analysis; in Section 3 the main characteristics of the MSA-BP, namely, the input matching, radiation characteristics, and efficiency, both in free space and in the vicinity of the human body are presented; finally the on-body propagation performance between two MSA-BPs placed on an arm phantom are numerically studied and compared with measurements with a human subject in an anechoic chamber. These results are also compared with those of another MSA design without backplane in order to demonstrate the benefits of the MSA$\mathrm{BP}$, in particular with regards to the significant improvement (about $15 \mathrm{~dB}$ ) of the channel path loss (PL) along a human arm.

\section{Ultra-Wideband Multislot Antenna Design}

After the abovementioned requirements, the design objectives have been selected as follows:

(i) operation over the lowest UWB sub-band,

(ii) planar geometry, tangent to the body,

(iii) single etching layer,

(iv) thickness as small as possible,

(v) reduction of body proximity effects, in particular losses,

(vi) improvement of the radio link budget along the body.

A slot type antenna was consequently chosen, designed and prototyped using a desensitization technique. The overall size of the proposed antenna is $68.1 \times 41.98 \times$ $4.445 \mathrm{~mm}^{3}$. The MSA-BP is fed by a tapered CPW line, which provides wideband matching [27]. It consists of two layers: the slot is etched in the ground plane on the top substrate (Arlon AD1000, $\varepsilon_{r}=10.2$ ), and a second substrate (Neltec, $\left.\varepsilon_{r}=2.33\right)$ is used to place the backplane reflector at a convenient distance from the slot plane. The antenna geometry and parameters are shown in Figure 1. The backplane has the size of the substrate $\left(68.1 \times 41.98 \mathrm{~mm}^{2}\right)$. The distance $t\left(t=t_{s 1}+t_{s 2}\right.$, Figure 1) between the slot layer and the backplane has a significant influence on the antenna input impedance.

In order to minimize the MSA-BP thickness, an RF substrate has been used to put away the backplane, instead of resorting to a foam spacer, which has two drawbacks: increase the thickness (as in [23], with a thickness of $7.58 \mathrm{~mm}$ 


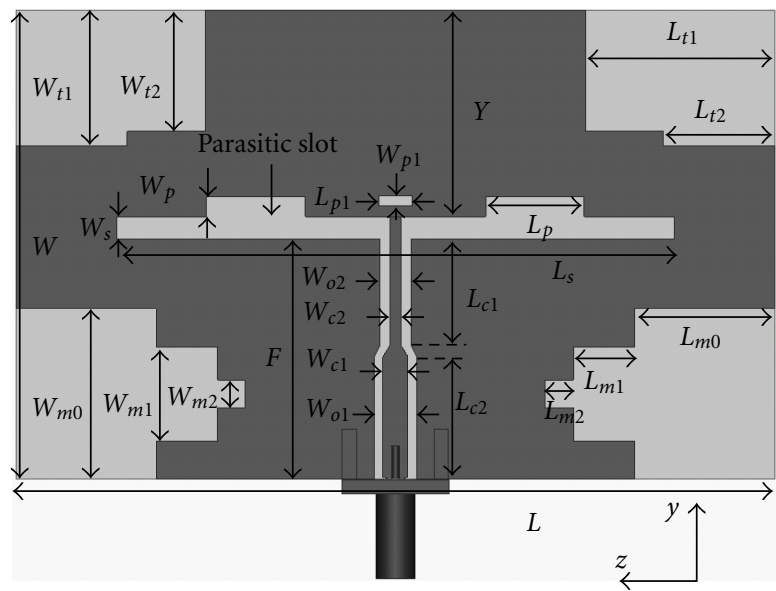

(a) MSA-BP top view

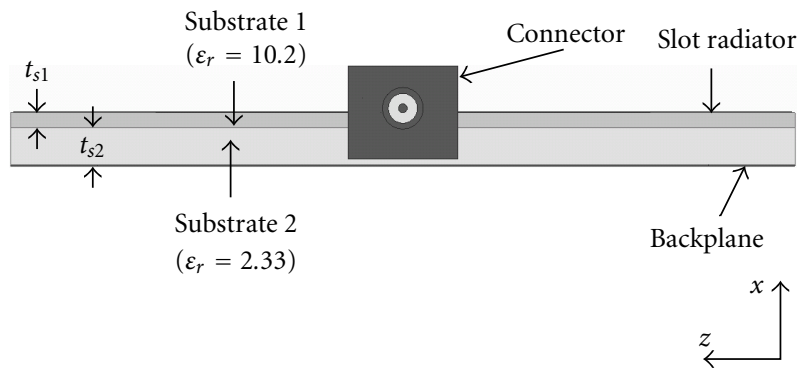

(b) MSA-BP side view

Figure 1: Geometry of the MSA-BP.

TABLE 1: Design parameters of the MSA-BP (mm).

\begin{tabular}{|c|c|}
\hline Parameters & Values \\
\hline$L$ & 68.1 \\
\hline$W$ & 42.1 \\
\hline$F$ & 21.55 \\
\hline$Y$ & 18.54 \\
\hline$L_{s}$ & 50 \\
\hline$W_{s}$ & 2 \\
\hline$L_{p}$ & 8.84 \\
\hline$W_{p}$ & 1.8 \\
\hline$L_{p 1}$ & 3 \\
\hline$W_{p 1}$ & 0.9 \\
\hline$L_{t 1}$ & 17 \\
\hline$W_{t 1}$ & 12.18 \\
\hline$L_{t 2}$ & 10 \\
\hline$W_{t 2}$ & 10.88 \\
\hline$L_{m 0}$ & 12.6 \\
\hline$W_{m 0}$ & 15.31 \\
\hline$L_{m 1}$ & 5.5 \\
\hline$W_{m 1}$ & 8.4 \\
\hline$L_{m 2}$ & 2.5 \\
\hline$W_{m 2}$ & 2.5 \\
\hline$L_{c 1}$ & 9.55 \\
\hline$L_{c 2}$ & 11 \\
\hline$W_{c 1}$ & 2.25 \\
\hline$W_{c 2}$ & 1 \\
\hline$W_{o 1}$ & 3.8 \\
\hline$w_{o 2}$ & 2.8 \\
\hline$t_{s 1}$ & 1.27 \\
\hline$t_{s 2}$ & 3.175 \\
\hline
\end{tabular}

for a higher operating frequency band) and make manufacturing more difficult to industrialize (as foam metallization is more complicated).
To understand the influence of the antenna design parameters on its impedance bandwidth, a systematic parametric analysis is performed. The final "optimal" values are summarized in Table 1.

2.1. Parametric Analysis of the Effect of the Parasitic Slots. The function of the two "parasitic slots" $\mathrm{PS}_{1}$ and $\mathrm{PS}_{2}$ is to improve the impedance matching over the operating frequency band. Their width, $W_{p}$ in particular, has a significant influence over the full band. The effect on the reflection coefficient $\left|S_{11}\right|$ is presented for $W_{p}$ varying from 0.6 to $1.8 \mathrm{~mm}$ (Figure 2). As can be observed, increasing $W_{p}$ improves the matching, particularly over the upper band from 4.0 to $6.0 \mathrm{GHz}$. A good result, compliant with the objectives, can be achieved for the "optimized" value of $W_{p}=1.8 \mathrm{~mm}$.

2.2. Analysis of the Main Slot Positioning. The position of the main slot, controlled by $F$ and $Y$ (Figure 1(a)), has a significant effect over the entire operating frequency band. The distance $F$ between the slot and the bottom edge of the substrate, mainly affects the upper part of the band (4.1$6.0 \mathrm{GHz}$ ), whereas the distance $Y$ between the upper edge of the substrate and the slot, mainly controls the lower band (3.4-4.3 GHz).

Figure 3 presents the influence of the $(F, Y)$ couple on the MSA-BP reflection coefficient. The two resonances in the lower frequency band $(3.4-4.5 \mathrm{GHz})$ are shifted up as $F$ increases. Simultaneously, as $Y$ decreases, the impedance matching over the higher band $(5.0-6.0 \mathrm{GHz})$ is improved. It should be noticed that when $F$ increases, the return loss from 5.0 to $6.0 \mathrm{GHz}$ is degraded, but this effect can be correctly compensated by conveniently adjusting the value of $Y$. The reverse also occurs for the lower band, so that the two parameters have interplay compensation roles and should be consequently jointly optimized.

2.3. Influence of the Space between the Slot and the Backplane. As the backplane acts as a reflector, its distance from the 


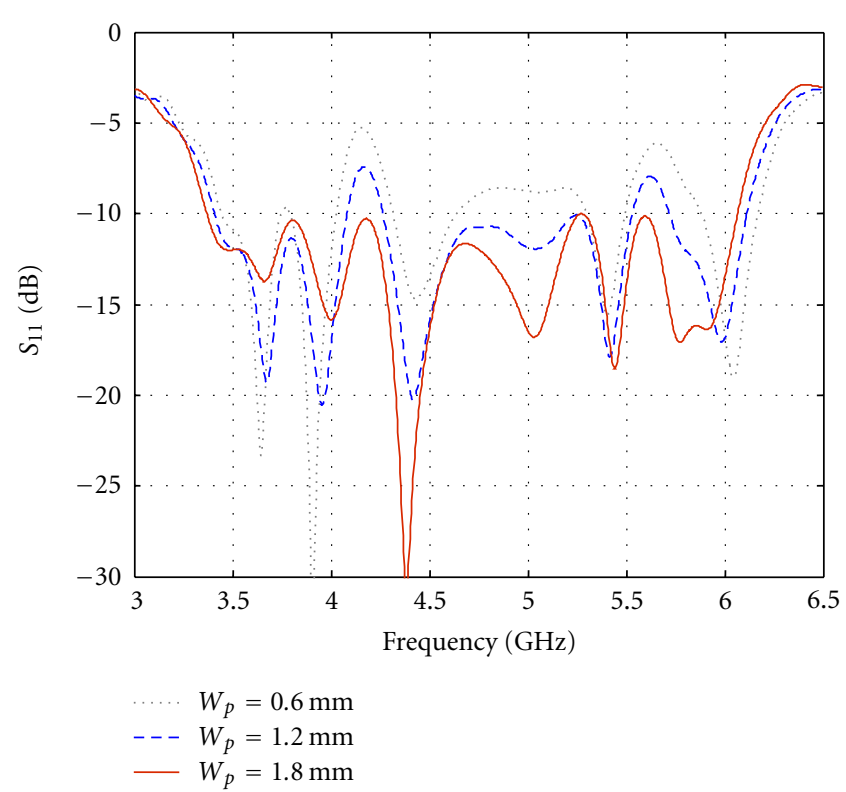

Figure 2: Reflection coefficient of the MSA-MP for different $W_{p}$.

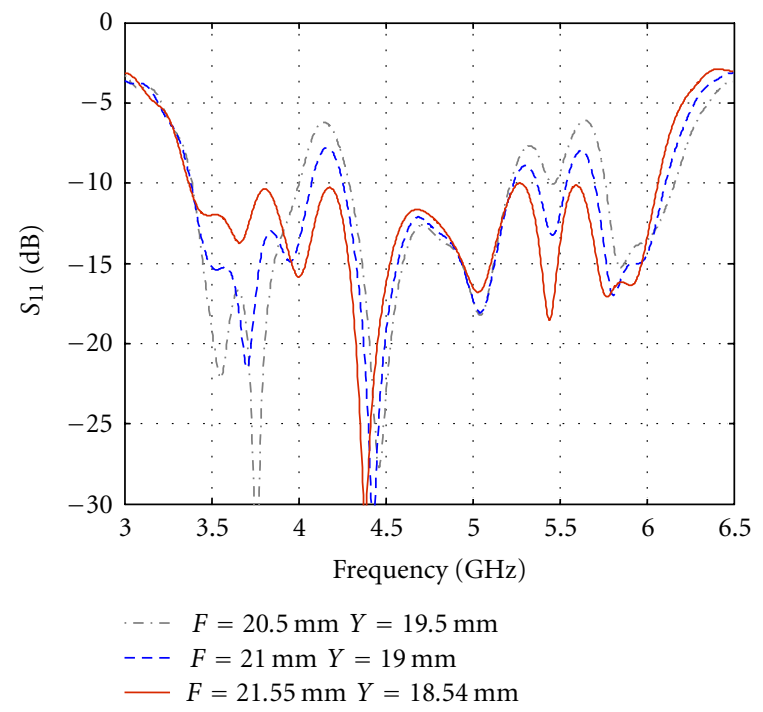

Figure 3: Reflection coefficient of the MSA-BP for different $(F, Y)$ couples.

slot should be finely tuned, because its effect on the input impedance is frequency dependent for obvious reasons.

Figure 4 shows the $\left|S_{11}\right|$ of the MSA-BP for different reflector-slot spacing $t$ (adjusting $t_{s 2}$ and keeping $t_{s 1}$ constant). The simulated relative input bandwidth for a spacing of $t=4.445 \mathrm{~mm}$ is about $56.9 \%$. The antenna proposed in [23] presents a bandwidth of $47 \%$ for a spacing of $6 \mathrm{~mm}$, whereas in [25] a bandwidth of $18 \%$ is achieved for a spacing of $16.6 \mathrm{~mm}$. By comparison, the MSA-BP has a wider impedance bandwidth for a smaller thickness.

2.4. Impedance Matching for the Lower Frequency Band (3.4$4.5 \mathrm{GHz}$ ). A pair of tapered slots, with the purpose of

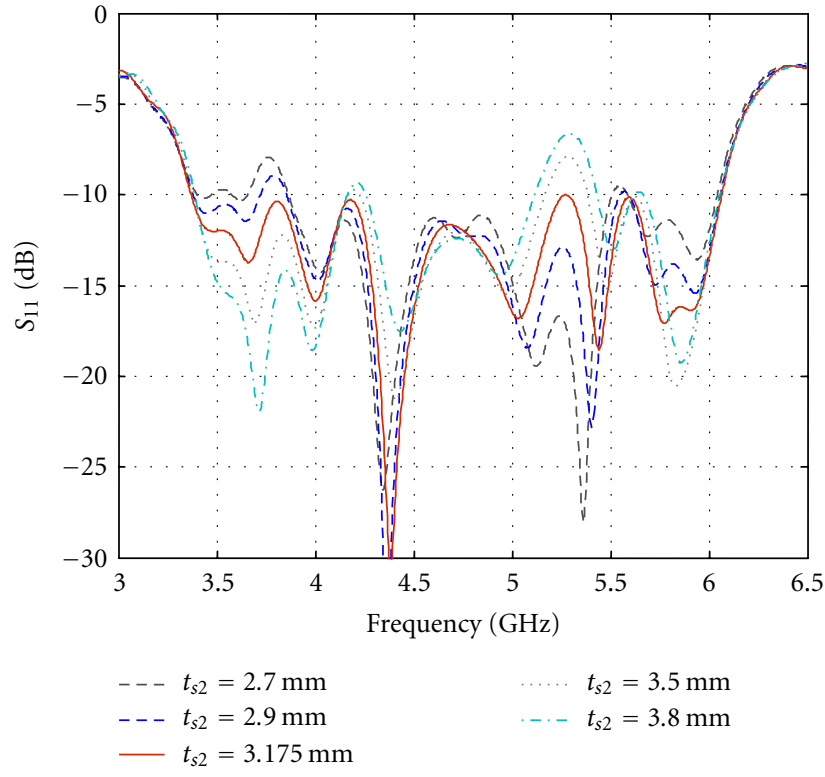

FIGURE 4: Reflection coefficient of the MSA-BP for different thicknesses $t$.

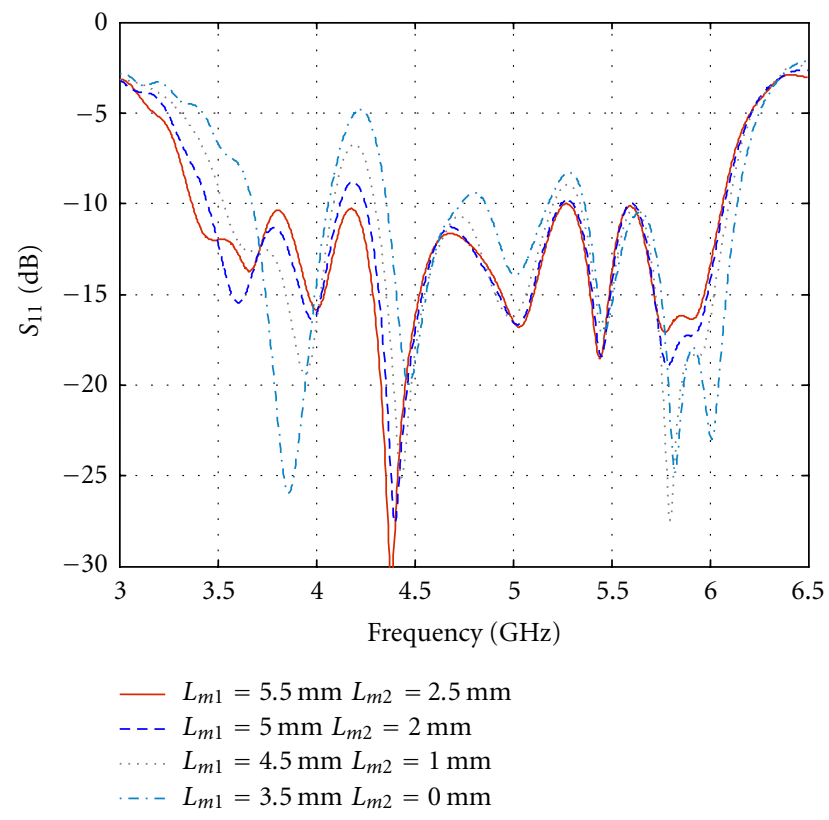

FIGURE 5: Reflection coefficient of the MSA-BP for different $L_{m 1}$ and $L_{m 2}$ combinations.

achieving a good impedance matching for the lower frequency band $(3.4-4.5 \mathrm{GHz})$, was etched on the two bottom corners of the ground plane (Figure 1). The return loss of the MSA-BP, when varying their lengths $\left(L_{m 1}, L_{m 2}\right)$, was investigated.

Figure 5 shows the $\left|S_{11}\right|$ of the MSA-BP for different $L_{m 1}, L_{m 2}$ combinations. The impedance matching is greatly improved in the lower band (3.4 to $4.5 \mathrm{GHz}$ ) when the values of $L_{m 1}$ and $L_{m 2}$ decrease, whereas the high frequencies $(4.5-6.07 \mathrm{GHz})$ are weakly affected by these parameters. 
The slots width $\left(W_{m k}\right)$ has almost no effect on the input impedance.

2.5. Impedance Matching for the Central Frequency Band (4.3$5.5 \mathrm{GHz})$. The upper tapered slots etched in the ground plane (Figure 1) are devoted to the matching of the central frequency band $(4.3-5.5 \mathrm{GHz})$. The widths of these matching slots are, respectively, denoted $W_{t 1}$ and $W_{t 2}$. Figure 6 shows the $\left|S_{11}\right|$ for different values of them. The effect on the lower and higher bands is negligible. An optimal result is obtained for $\left(W_{t 1}, W_{t 2}\right)=(10.885,1.3) \mathrm{mm}$. The lengths $L_{t 1}$ and $L_{t 2}$ have only a slight effect on the whole operating band.

2.6. Reference Antenna Design: Multislot Antenna without Backplane. In order to underline the desensitization characteristics of the MSA-BP, another multislot antenna (taken as a "reference") without any metallic backplane was designed. The design of this reference MSA is actually based on that of the MSA-BP. Its geometry is shown in Figure 7, with an overall size of $56 \times 28.25 \times 1.524 \mathrm{~mm}^{3}$. It was realized on a $1.524 \mathrm{~mm}$ thick Neltec substrate $\left(\varepsilon_{r}=3.48\right)$. It presents an operating bandwidth of 3-6 GHz.

\section{Comparison of Antennas Operating Near a Human Body Phantom}

3.1. Human Body Model for Numerical Electromagnetic Simulations. As we know, in WBAN applications, a strong coupling effect takes place between the reactive field of the antenna and the human body, which can modify substantially its impedance and radiation characteristics. Therefore, it is necessary to take into account the human body proximity from the very beginning of the design process, and in particular in the EM simulations. A dispersive human arm phantom is modeled as a trilayer dielectric in CST, corresponding to tissues of skin ( $1 \mathrm{~mm}$ thick), fat ( $3 \mathrm{~mm}$ thick), and muscles ( $40 \mathrm{~mm}$ thick), which is a good compromise between model complexity and representativeness of the dielectric effects of the human body for "exterior problems." The arm phantom is a square section cylinder with overall dimension of $400 \times 70 \times 44 \mathrm{~mm}^{3}$. The values of the permittivities and conductivities of the considered tissues were extracted from $[28,29]$ and fitted with a 2nd-order model. The configuration used to simulate the MSA-BP mounted on the arm phantom is shown in Figure 8 . As the proximity of the human body influences the current distribution of the slot radiator, for a fair comparison between both antennas (MSA-BP/MSA), the spacing between any antenna and the phantom or human subject is defined here as the distance $d$ between the radiator layer and the body (See Figures 8 and 15).

3.2. Reflection Coefficient. Two prototypes were realized with a mechanical drilling tool. As an over etching has been observed, a retro-simulation has been performed with the true prototypes dimensions to take into account this fabrication defect as well as the well-known "cable effect" (as the antenna is not strictly balanced). In addition, better

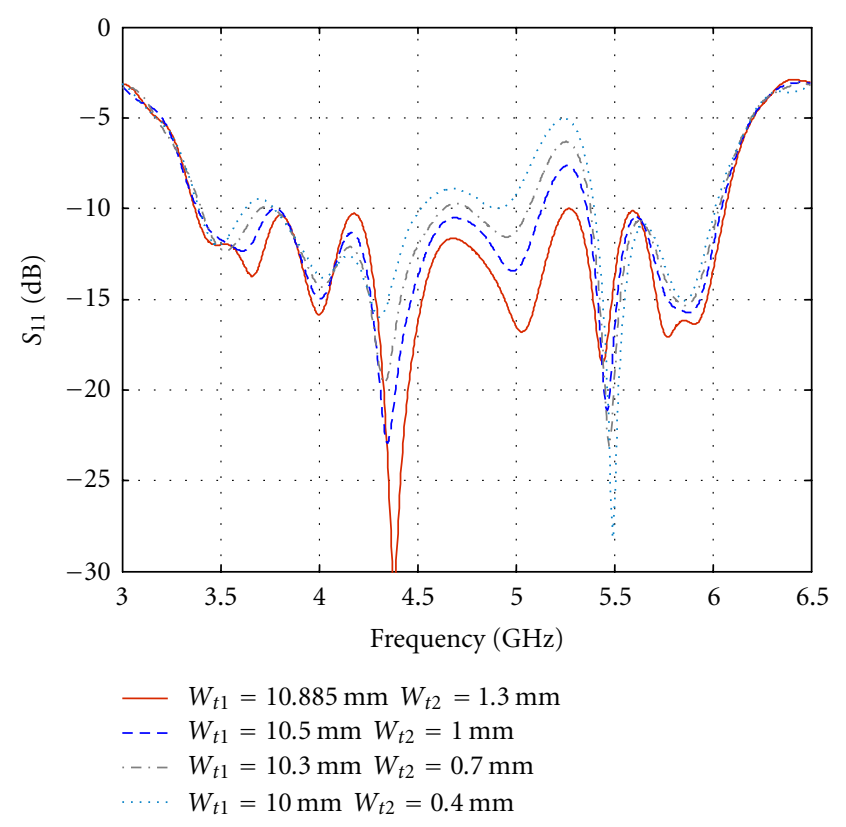

FIGURE 6: Reflection coefficient of the MSA-BP for different $W_{t 1}$ and $W_{t 2}$ combinations.

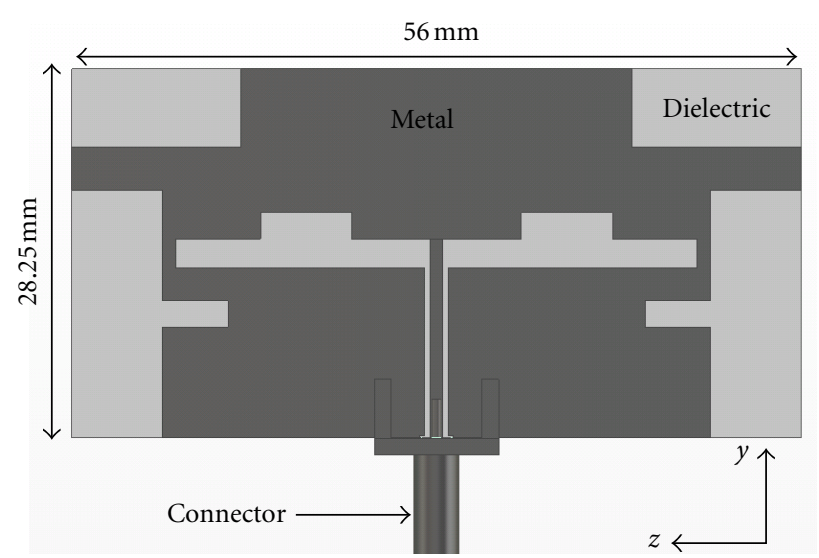

FIGURE 7: Geometry of the reference MSA antenna without metallic backplane.

simulation settings providing an improved accuracy were used (finer meshing, convergence criteria more stringent, etc.). Although the agreement between simulation and measurement remains moderate (Figure 9), it is clearly improved compared with the initial simulation. Discrepancies in particular in the low frequency range are still significant. However, we have observed for many years that a good agreement between simulations and measurements for UWB antennas neither balanced nor clearly grounded is often difficult to obtain in particular in the low frequency range. Nevertheless, on the whole the input bandwidth provided by the simulation is essentially correct.

The effect of the body is analyzed, comparing the MSABP behavior either in free space or in the vicinity of a human arm (Figure 9). The distance between the slot layer 


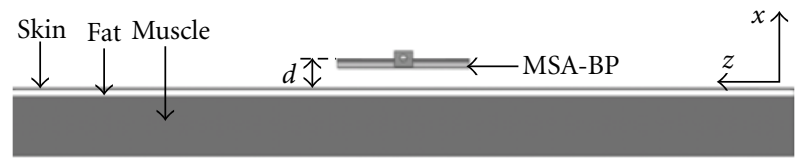

FIGURE 8: Configuration of the MSA-BP on a trilayer arm phantom.

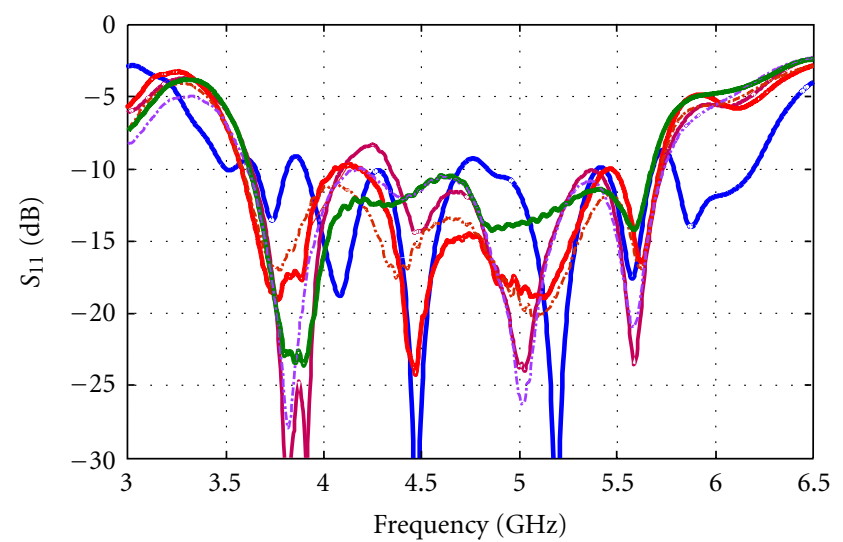

Retro-simulation $\left(t_{s 1}=1.2 \mathrm{~mm}, t_{s 2}=3.1 \mathrm{~mm}\right.$,
cable length $=50 \mathrm{~mm})$
- MSA-BP1 (measured, isolated)
- - MSA-BP2 (measured, isolated)
-.- MSA-BP2 (measured with ferrite beads, isolated)
- MSA-BP1 (measured with ferrite beads, isolated)

FIGURE 9: Simulated and measured reflection coefficient of the MSA-BP, either isolated or on a human arm (male, $1.75 \mathrm{~m}$ and $75 \mathrm{~kg})$.

and the arm is chosen here as $d=6.5 \mathrm{~mm}(\sim t+2 \mathrm{~mm})$, corresponding roughly to the thickness of a cloth. Figure 10 presents the measured reflection coefficient of the MSA-BP for different distances $d$ from a human subject (male, $1.75 \mathrm{~m}$ and $75 \mathrm{~kg}$ ). We can see that the $\left|S_{11}\right|$ is almost insensitive to this distance, at least down to $5.5 \mathrm{~mm}$. It can be observed that the desensitization property has been efficiently achieved, as the human arm has a moderate global effect on the matching over the whole band.

For comparison purpose, the measured reflection coefficient of the reference MSA in the same experimental conditions is shown in Figure 11. As expected, the significant effects on the $\left|S_{11}\right|$ described in the introduction can be observed, notably the fall of the low matching frequency as well as the shifts of the resonance frequencies. We can also observe significant effects on its simulated radiation efficiency (Table 2), resulting from the absorption, reflection, and diffraction of the arm phantom.

\subsection{Radiation Patterns}

3.3.1. Simulated and Measured Realized Gain Patterns of the MSA-BP. The comparison between the measured and the simulated copolar ( $\varphi$-component) realized gain patterns (Figures 12(a)-12(f)) for two principle planes (azimuth and

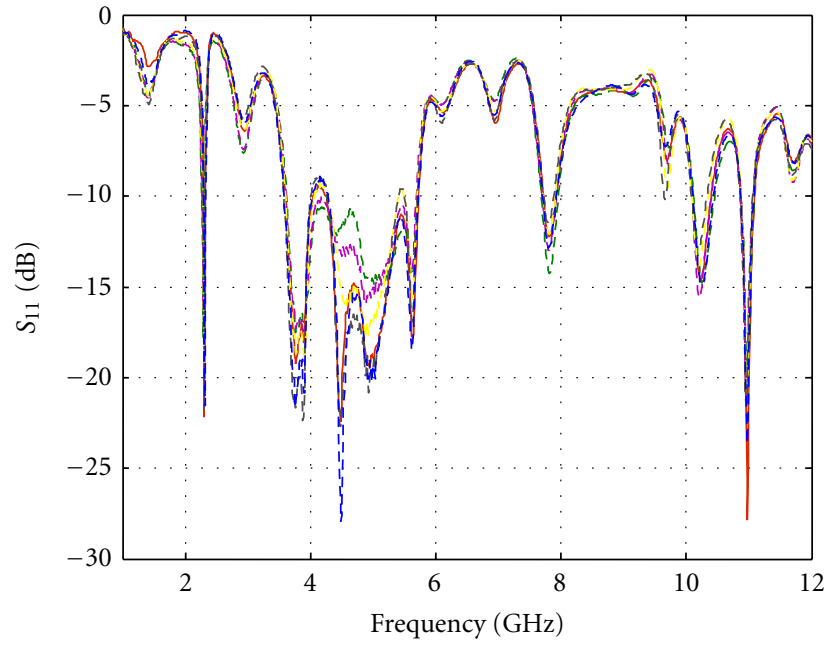

$$
\begin{array}{ll}
\text { In free space } & d=9.5 \mathrm{~mm} \\
---d=5.5 \mathrm{~mm} & --d=14.5 \mathrm{~mm} \\
---d=7.5 \mathrm{~mm} & ---d=24.5 \mathrm{~mm}
\end{array}
$$

FIGURE 10: Measured reflection coefficient of the MSA-BP on a human subject for different values of the separation distance $d$.

TABLE 2: Radiation efficiency of the MSA-BP and the reference MSA.

\begin{tabular}{lccc}
\hline \multicolumn{4}{c}{ Radiation efficiency of MSA-BP/MSA (\%) } \\
Frequency $(\mathrm{GHz})$ & \multicolumn{3}{c}{ Antenna/phantom spacing $d(\mathrm{~mm})$} \\
& 6.5 & 7.5 & 8.5 \\
\hline 3.5 & $47 / 25$ & $49 / 29$ & $51 / 33$ \\
4.5 & $62 / 26$ & $62 / 33$ & $62 / 39$ \\
5.5 & $51 / 46$ & $55 / 54$ & $59 / 62$ \\
\hline
\end{tabular}

elevation normal to the antenna plane) at 4,5 , and $6 \mathrm{GHz}$, respectively, is presented in Figure 12. The discrepancies observed in the elevation plane for angles near $180^{\circ}$ are due to the "shadowing" effect of the positioner head in a "rear solid angle." The measured and simulated Mean Realized Gains $(M R G,(1),[5,6])$ of the MSA-BP are presented in Figures $12(\mathrm{~g})-12(\mathrm{i})$, for three principal plane cuts, as well as a $3 \mathrm{D}$ pattern in Figure $12(\mathrm{j})$. Its definition is reminded below:

$$
M R G^{\varphi}=\frac{1}{B W} \int_{f_{1}}^{f_{2}} G_{r}^{\varphi}(f, \hat{\mathbf{r}}) d f
$$

where $B W$ is the antenna operating bandwidth $\left(f_{1}, f_{2}\right), G_{r}^{\varphi}$ is the copolar realized gain and is the radial unit vector. The broadside realized gain $\left((\theta, \varphi)=\left(90^{\circ}, 0\right)\right)$ against frequency is shown in Figure $12(\mathrm{k})$.

3.3.2. Simulated Realized Gain Patterns of the MSA-BP on the Arm Phantom. The copolar realized gain patterns of the MSA-BP in the $x \mathrm{O} y\left(\theta=90^{\circ}\right), x \mathrm{O} z(\varphi=0)$ and $y \mathrm{O} z$ $\left(\varphi=90^{\circ}\right)$ planes were calculated at three frequency points $(4,5$ and $6 \mathrm{GHz})$ for three situations: antenna in free space, and antenna close to the phantom, 6.5 and $9.5 \mathrm{~mm}$ away. 


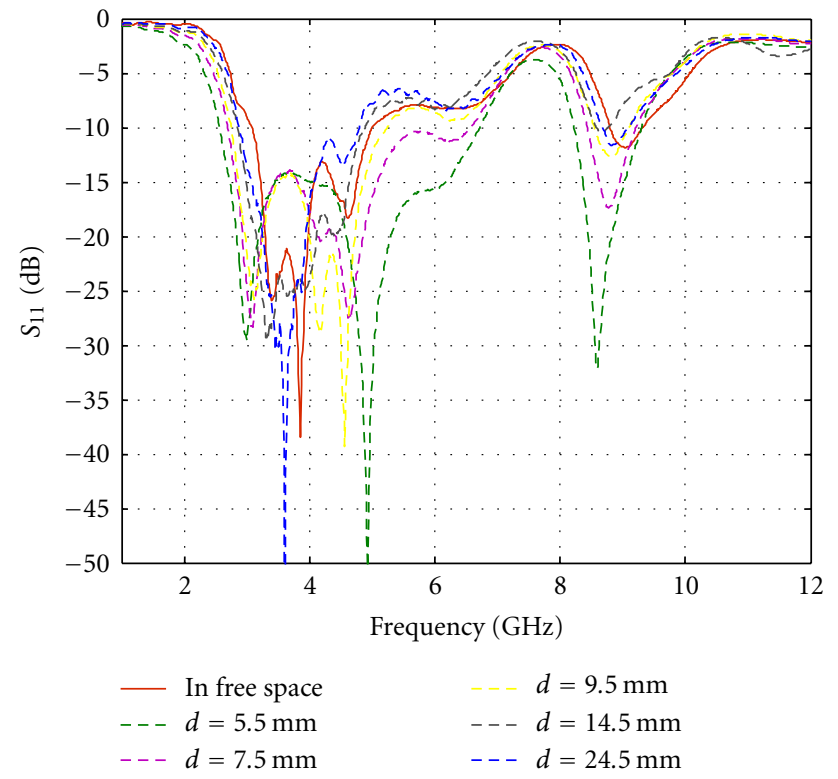

Figure 11: Measured reflection coefficient of the reference MSA on a human subject for different values of the separation distance $d$.

TABLE 3: SAR values of MSA-BP and reference MSA (input power $\left.P_{\text {in }}=1 \mathrm{~W}\right)$.

\begin{tabular}{lcc}
\hline Frequency $(\mathrm{GHz})$ & \multicolumn{2}{c}{ SAR $(\mathrm{W} / \mathrm{kg})$} \\
& MSA-BP & Reference MSA \\
\hline 3.5 & 4.50 & 29.36 \\
4.5 & 4.10 & 25.02 \\
5.5 & 6.06 & 16.88 \\
\hline
\end{tabular}

We can observe from Figure 13 that the arm phantom has a moderate effect on the radiation characteristics of the MSA$\mathrm{BP}$, in particular in the main lobe, apart at $6 \mathrm{GHz}$ in the elevation plane, for which the reflector effect of the body compensates the main lobe splitting in free space (due to an "array effect" which can be observed on the current surface density). Globally, the most significant effect is typically restricted to the back hemisphere. From Figure 13(j), it can be seen that the proximity of the human body has almost no effect on the energy transfer in the antenna plane $(y \mathrm{O} z)$, particularly along the slot (directions around $\theta=0^{\circ}$ and $180^{\circ}$ ). The $M R G$-although relatively low as expectedremains satisfactory $(\sim[-8,-5] \mathrm{dBi})$ in this plane over a wide beam $\left(\sim 120^{\circ}\right)$ around $-90^{\circ}$.

3.4. Radiation Efficiency and SAR. This section is devoted to the investigation of losses. The radiation efficiency for both MSA-BP and reference MSA, either in free space or in the vicinity of the body is analyzed numerically. Both metal (copper, with a conductivity of $\sigma_{\mathrm{Cu}}=5.96 \times 10^{7} \mathrm{~S} / \mathrm{m}$ ) and substrate losses $(\tan \delta=0.003)$ are considered in the simulations, as well as one arm phantom model, involving the three-layer model above mentioned. Three values (6.5,
7.5 , and $8.5 \mathrm{~mm}$ ) of the spacing $d$, for studying the sensitivity to the body proximity of both antennas, are considered.

Table 2 shows the overall radiation efficiency of both antennas near the arm phantom model. The efficiency of the MSA-BP is $47 \%$ at $3.5 \mathrm{GHz}$ for a separation distance of $6.5 \mathrm{~mm}$, whereas it falls to only $25 \%$ for the reference MSA, which gives an improvement of $22 \%$ for the MSABP. Common UWB antennas-in particular nor clearly grounded nor well balanced, such as planar monopole-like ones [15-17] - are sensitive to the distance between the sources (currents) and the phantom. For example, in Table 2 the variations of the radiation efficiency are $8 \%, 13 \%$, and $16 \%$ at $3.5,4.5$, and $5.5 \mathrm{GHz}$, respectively, for the reference MSA, whereas for the MSA-BP the variations are only 4, 0 and $8 \%$ at $3.5,4.5$, and $5.5 \mathrm{GHz}$, respectively. The MSA-BP is almost insensitive to the distance between the radiation layer and the phantom, which is an important concern for antennas used in WBANs [13].

The radiation efficiency of the MSA-BP has been significantly improved as compared to that of the reference MSA. As will be shown hereafter, this reduction of losses in the reactive near field region plays a significant role in the improvement of the PL in any on-on radio links, which is actually the ultimate goal.

The power absorption of the three-layer arm phantom is studied in the skin layer (where the maximum absorption occurs) [30]. The configuration of the simulation is shown in Figure 8. Figure 14 shows the distribution of the normalized power in the skin layer for both antennas. The power absorption for the MSA-BP occurs near the backplane edges (appearing as a sort of diffraction effect) and is, as expected, far less than its counterpart for the reference MSA.

To confirm more thoroughly these results, Figure 15 shows the SAR (specific absorption rate, averaged over $10 \mathrm{~g}$ ) distribution for both antennas at 3.5, 4.5, and 5.5 GHz. The three-layer phantom model is used in these simulations. The distance between the radiation layer and the phantom is set to $6.5 \mathrm{~mm}$. The maximal SAR values (for an input power of $P_{\text {in }}=1 \mathrm{~W}$ ) for both MSA-BP and MSA are shown in Table 3. The screening effect of the MSA-BP reduces considerably the energy absorbed by the body in comparison with the reference MSA, SAR values being reduced typically by a factor of 3 to 6 . In addition, the SAR of the MSABP depends weakly on the frequency, which confirms the results reported in Table 2, showing respectively high and moderately frequency-dependent values of the radiation efficiency.

3.5. Path Gain. With the purpose of examining the propagation performance of the MSA-BP along the body, a simple radio link scenario along the arm is considered, both in simulation (with the phantom) and with a human subject. Two antennas (either MSA-BP or MSA) are placed tangentially to the body, $180 \mathrm{~mm}$ apart (Figure 16(a)). Several values (6.5, $9.5,11.5,14.5,19.5$, and $24.5 \mathrm{~mm}$ ) of the spacing $d$ are considered. Foam spacers are used for the measurement. 


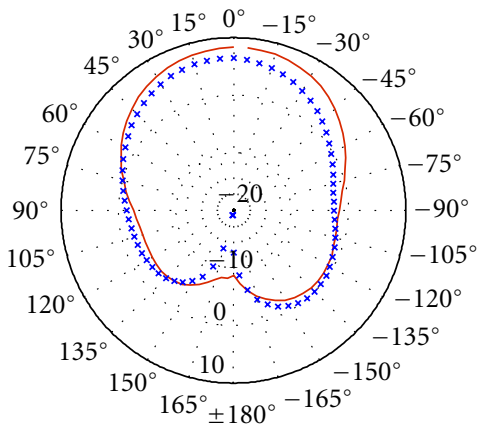

(a) $x \mathrm{O} y 4 \mathrm{GHz}$

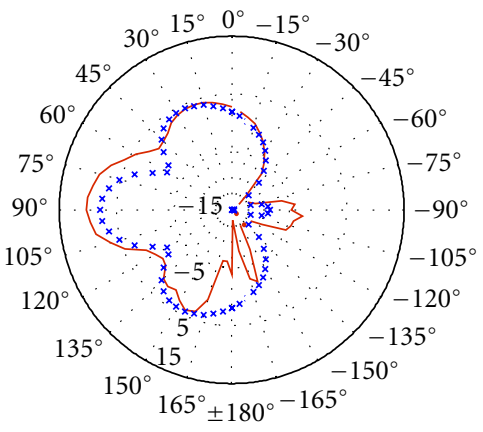

(d) $x \mathrm{O} z 5 \mathrm{GHz}$
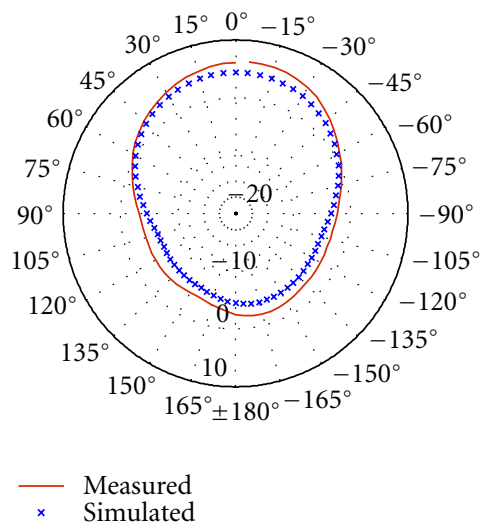

(g) $M R G \_x \mathrm{O} y$

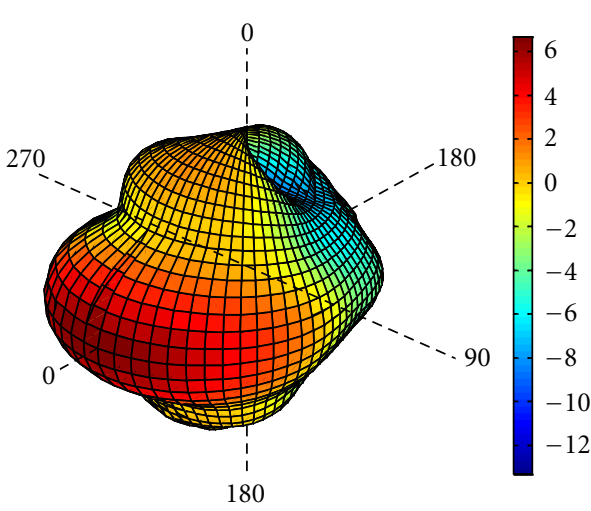

(j) $3 \mathrm{D} M R G$ (over $\sim 3-6 \mathrm{GHz}$ )

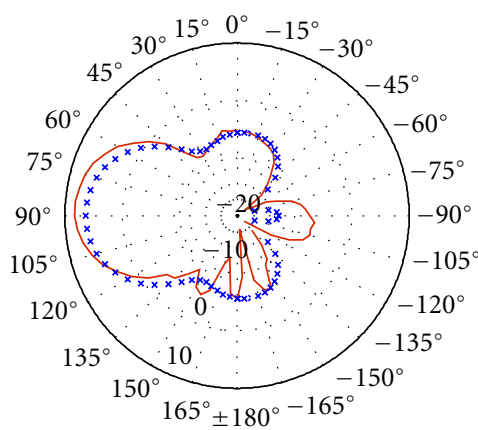

(b) $x \mathrm{O} z 4 \mathrm{GHz}$

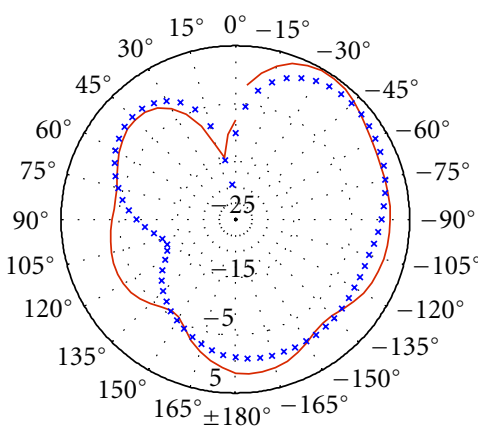

(e) $x \mathrm{O} y 6 \mathrm{GHz}$

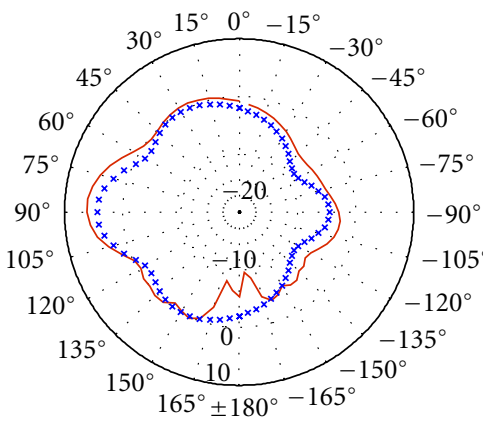

$\begin{array}{ll}\text { Measured } \\ \times & \text { Simulated }\end{array}$

(h) $M R G_{-} x \mathrm{O} z$

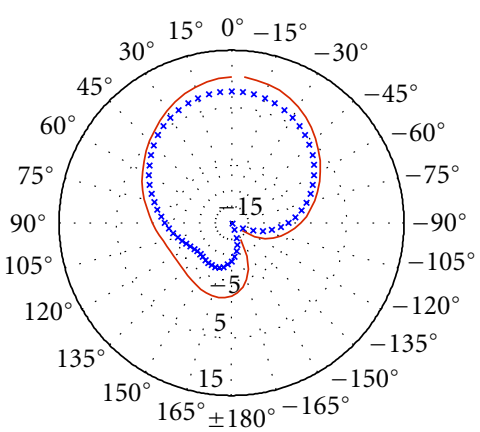

(c) $x \mathrm{O} y 5 \mathrm{GHz}$

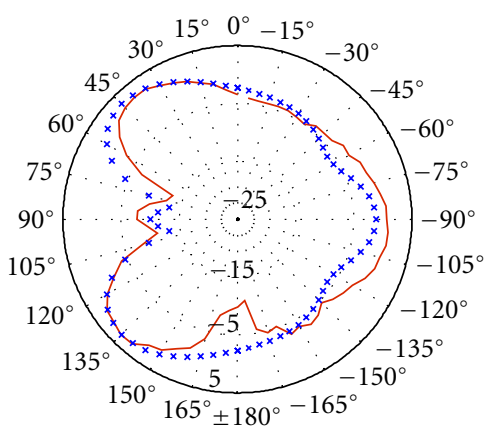

(f) $x \mathrm{O} z 6 \mathrm{GHz}$

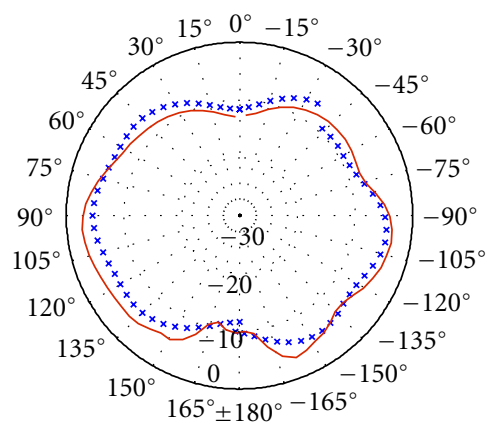

- Measured

(i) $M R G \_y \mathrm{O} z$

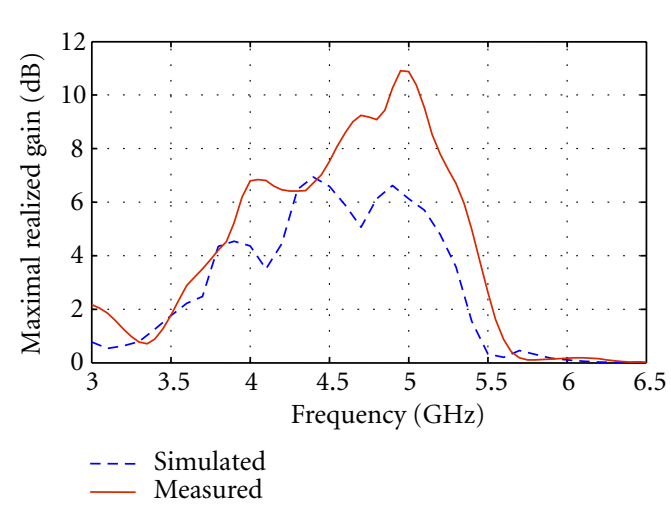

(k) Broadside realized gain at $\left(\theta=90^{\circ}, \varphi=0\right)$

Figure 12: (a)-(f) Measured and simulated realized gain patterns of the MSA-BP in two principle planes (azimuth and normal elevation), $(\mathrm{g})-(\mathrm{j})$ measured and simulated mean realized gain $(2 \mathrm{D})$ in three plane cuts and a $3 \mathrm{D}$ mean realized gain pattern, $(\mathrm{k})$ broadside realized gain at $\left(\theta=90^{\circ}, \varphi=0\right)$. 


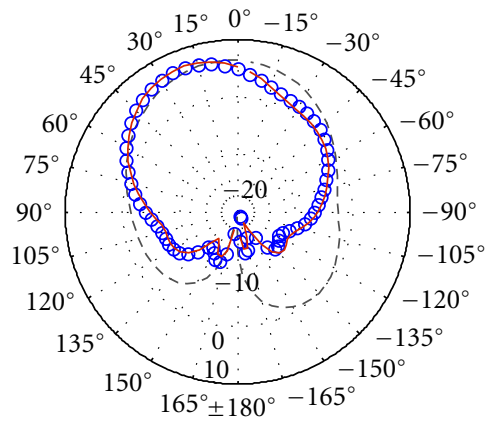

(a) $x \mathrm{O} y_{-} 4 \mathrm{GHz}$

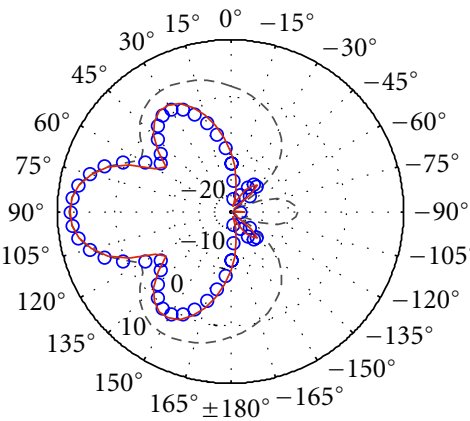

(d) $x \mathrm{O} z_{-} 5 \mathrm{GHz}$

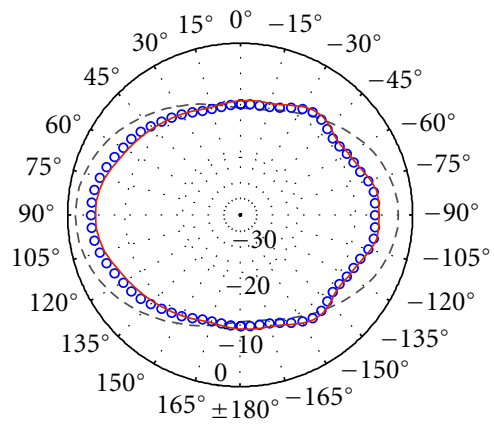

(g) $y \mathrm{O} z 4 \mathrm{GHz}$

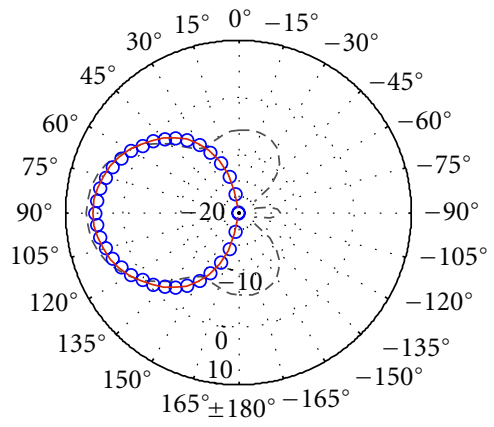

(b) $x \mathrm{O} z \_4 \mathrm{GHz}$

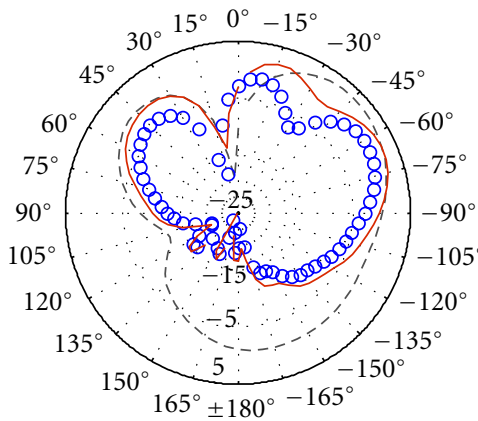

(e) $x \mathrm{O}_{-} \_6 \mathrm{GHz}$

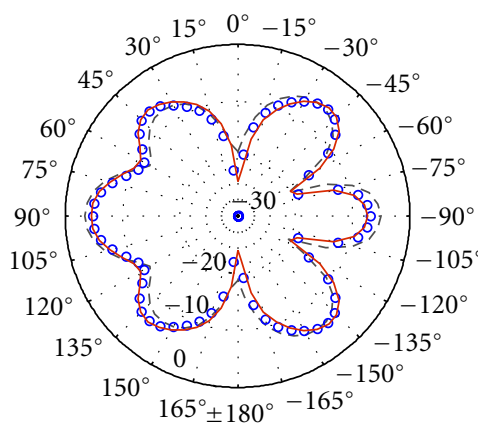

(h) $y \mathrm{O} z 5 \mathrm{GHz}$

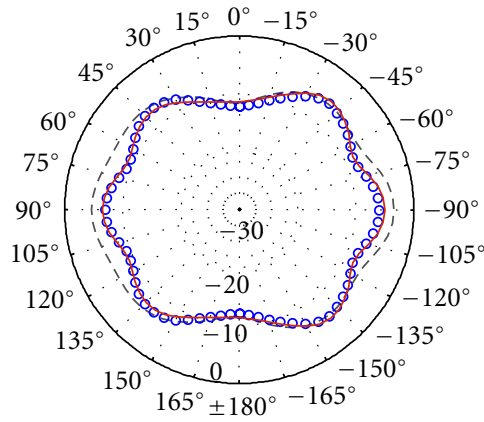

- - - MSA-BP in free space

- MSA-BP on phantom $(6.5 \mathrm{~mm})$

— MSA-BP on phantom $(9.5 \mathrm{~mm})$

(j) $y \mathrm{O} z M R G$

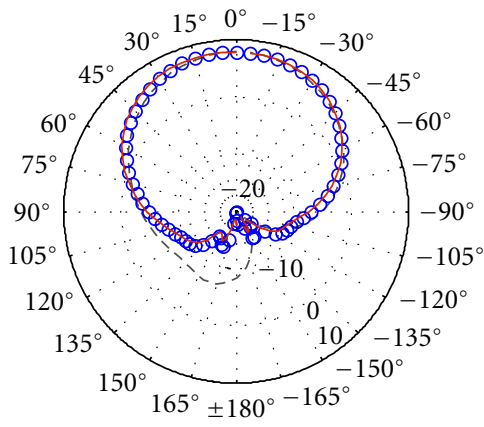

(c) $x \mathrm{O} y_{-} 5 \mathrm{GHz}$

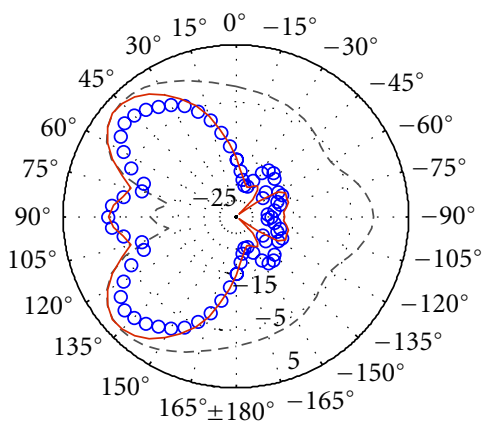

(f) $x \mathrm{O} z \_6 \mathrm{GHz}$

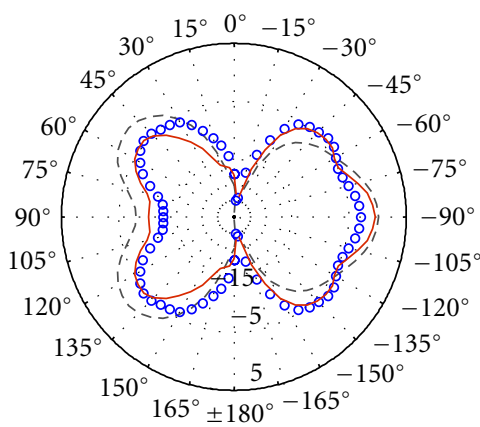

(i) $y \mathrm{Oz} 6 \mathrm{GHz}$

Figure 13: (a)-(i) Realized gain patterns of the MSA-BP in free space or close to an arm phantom in three plane cuts $\left(\left(\theta=90^{\circ}, \varphi=0, \varphi=\right.\right.$ $\left.90^{\circ}\right)$, (j) mean realized gain of the MSA-BP in the antenna plane $\left.y \mathrm{O} z\left(\varphi=90^{\circ}\right)\right)$. 


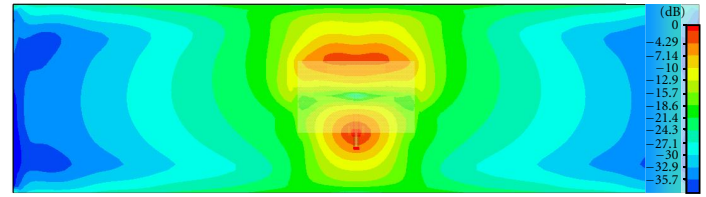

(a) MSA-BP at $4 \mathrm{GHz}$

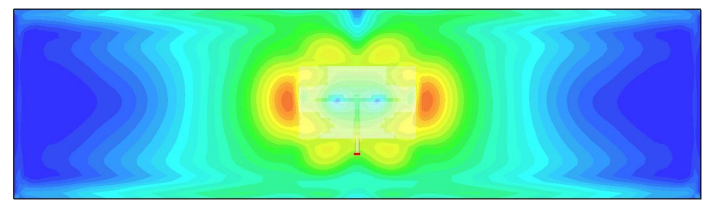

(c) MSA-BP at $5 \mathrm{GHz}$

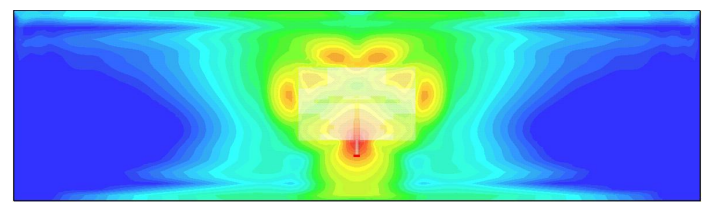

(e) MSA-BP at $6 \mathrm{GHz}$

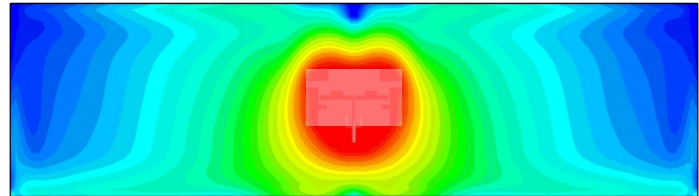

(b) Reference MSA at $4 \mathrm{GHz}$

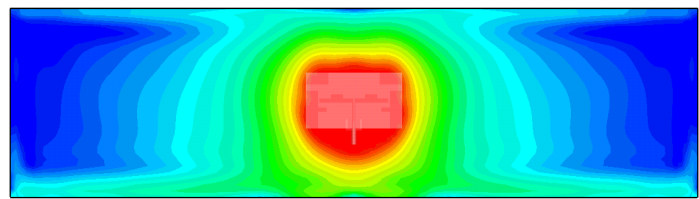

(d) Reference MSA at $5 \mathrm{GHz}$

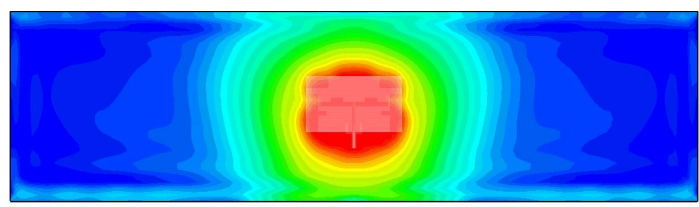

(f) Reference MSA at $6 \mathrm{GHz}$

FIgure 14: Power absorbed in the body, for the MSA-BP and the MSA.

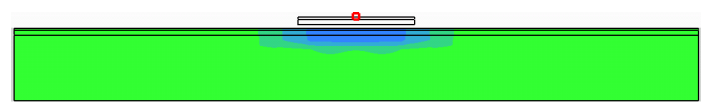

(a) MSA-BP at $3.5 \mathrm{GHz}$

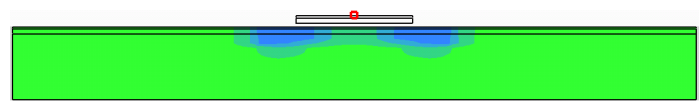

(c) MSA-BP at $4.5 \mathrm{GHz}$

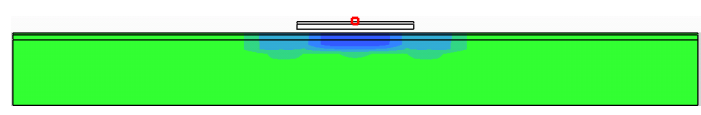

(e) MSA-BP at $5.5 \mathrm{GHz}$

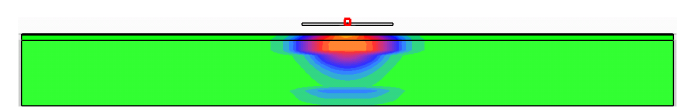

(b) Reference MSA at $3.5 \mathrm{GHz}$

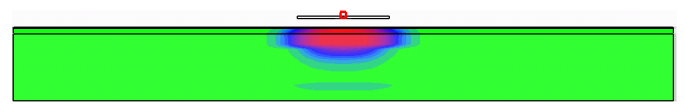

(d) Reference MSA at $4.5 \mathrm{GHz}$

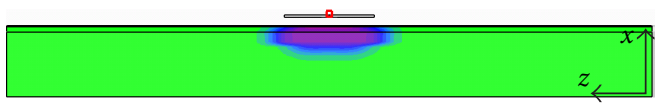

(f) Reference MSA at $5.5 \mathrm{GHz}$

$15 \mathrm{~W} / \mathrm{kg}$

FIGURE 15: SAR distribution of MSA-BP and reference MSA antenna (6.5 mm from radiation layer to phantom).

The global trends (Figure 16(b)) of both simulated and measured path gains (PG) are consistent. As expected, the PG is almost independent of the spacing $d$ with the MSABPs, whereas it is an increasing function of $d$ with the MSAs (only weakly in the measured case). The PG with the MSABPs is about $15 \mathrm{~dB}$ higher than that of the reference MSA.

\section{Conclusion}

A multislot antenna with a screening backplane (MSA-BP) for WBANs applications has been proposed. The MSA-BP has a good impedance matching from $3.38 \mathrm{GHz}$ to $6.07 \mathrm{GHz}$ and satisfies the UWB on-body propagation requirements. The design parameters of the MSA-BP and the screening effect of the backplane were studied carefully by means of EM simulations.
An arm phantom model with three layers (skin, fat, muscle) was used to examine the proximity effect of the human body on the characteristics of the MSA-BP. A reference MSA, without reflector, was designed to demonstrate the improved performance of the MSA-BP.

Results show that even if the distance between the radiation layer of the MSA-BP and the phantom is small, its impedance bandwidth is similar to that in free space, demonstrating the desensitization functionality. The radiation patterns of the MSA-BP are weakly affected by the body proximity in the main lobe. Its radiation efficiency in the vicinity of the body is significantly improved with respect to that of the reference MSA. For a simple scenario of radio link along the arm, this losses reduction corresponds to a PG higher by about $15 \mathrm{~dB}$. The screening effect also provides a benefit in terms of radiation exposure, the SAR of the MSA-BP being considerably reduced compared to that of the 


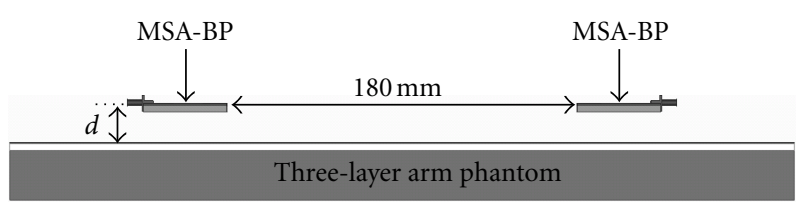

(a)

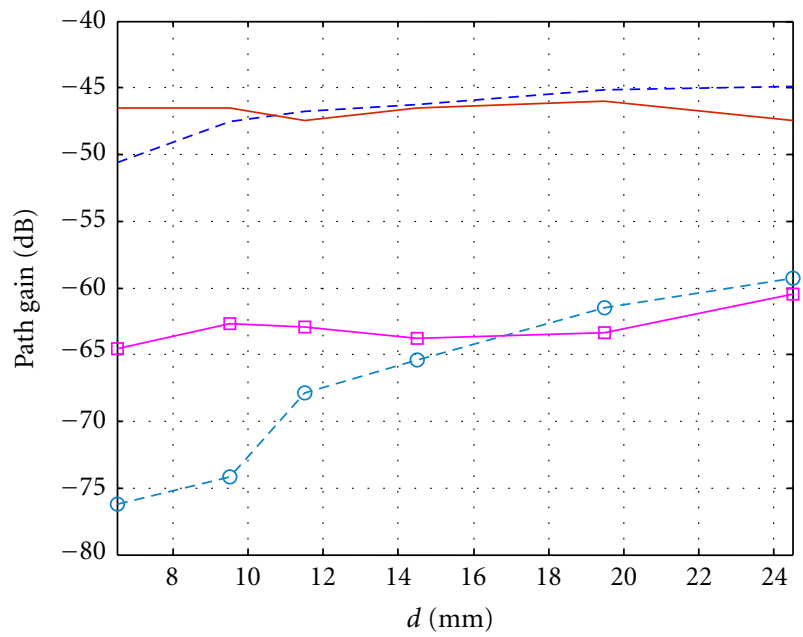

$\begin{array}{ll}--- & \text { MSA-BP (simulated) } \\ - & \text { MSA-BP (measured) } \\ -\ominus- & \text { Reference MSA (simulated) } \\ \square- & \text { Reference MSA (measured) }\end{array}$

(b)

FIgURE 16: (a) Transmission scenario on the arm phantom, (b) Simulated and measured path gain with 2 MSA-BPs (resp. MSAs) $180 \mathrm{~mm}$ apart on the arm.

reference MSA. In addition, the backplane effect increases the MSA-BP directivity, which is beneficial for on-off WBAN applications.

The desensitization concept can be applied as well to UWB (or NB) antennas for RFID tags in the microwave range. Indeed, these tags may be placed on many types of objects, including metallic, which may induce significant effects on their behavior.

\section{References}

[1] P. S. Hall and Y. Hao, Antennas and Propagation for Body Centric Communications Systems, Artech House, Norwood, Mass, USA, 2006.

[2] P. S. Hall and Y. Hao, "Antennas and propagation for body centric communications," in Procedings of the 1th European Conference on Antennas and Propagation (EuCAP '06), Nice, France, November 2006.

[3] T. Zasowski, F. Althaus, M. Staeger, A. Wittneben, and G. Troester, "UWB for noninvasive wireless body area networks: channel measure-measurements and results," in Proceedings of the IEEE Conference on Ultra Wideband Systems and Technologies (UWBST '03), Reston, VA, USA, November 2003.

[4] Federal Communication Commission, First Report and Order, February 2002.
[5] X. Begaud, Ed., Ultra Wide Band Antennas, John Wiley \& Sons, New York, NY, USA, 2011.

[6] C. Roblin, S. Bories, and A. Sibille, "Characterization tools of antennas in the Time Domain," in Proceedings of International Workshop on Ultra Wideband Systems (IWUWBS '03), Oulu, Finland, June 2003.

[7] X. H. Wu and Z. N. Chen, "Design and optimization of UWB antennas by a powerful CAD Tool: PULSE KIT," in Proceedings of the IEEE Antennas and Propagation Society International Symposium, pp. 1756-1759, June 2004.

[8] A. Sibille, "Role of joint antenna-channel dispersions on UWB energy capture in pulsed schemes," in Proceedings of the IEEE International Conference on Ultra-Wideband (ICUWB '06), pp. 207-211, Waltham, Mass, USA, September 2006.

[9] H. Schantz, The Art \& Science of Ultrawideband Antennas, Artech House, 2005.

[10] N. Chahat, M. Zhadobov, R. Sauleau, and K. Ito, "A compact UWB antenna for on-body applications," IEEE Transactions on Antennas and Propagation, vol. 59, no. 4, pp. 1123-1131, 2011.

[11] K. Yekeh Yazdandoost and R. Kohno, "UWB antenna for wireless body area network," in Proceedings of the Asia-Pacific Microwave Conference (APMC '06), pp. 1647-1652, December 2006.

[12] A. Lea, "Propagation between on-body antennas," IEEE Transactions on Antennas and Propagation, vol. 57, no. 11, pp. 36193626, 2009.

[13] C. Roblin, J. M. Laheurte, R. D’Errico et al., "Antenna design and channel modeling in the BAN context-part I: antennas," Annals of Telecommunications, vol. 66, no. 3-4, pp. 139-155, 2011.

[14] W. T. Chen and H. R. Chuang, "Numerical computation of human interaction with arbitrarily oriented superquadric loop antennas in personal communications," IEEE Transactions on Antennas and Propagation, vol. 46, no. 6, pp. 821-828, 1998.

[15] K. Y. Yazdandoost and R. Kohno, "The Effect of Human Body on UWB BAN Antennas," IEEE802. 15-07-0546-00-0ban.

[16] C. Roblin, "Parametric modeling of antennas influence on the path loss for UWB on-body WBAN scenarios," in COST 2100 Management Committee Meeting, pp. 23-25, Bologna, Italy, November 2010.

[17] Ch. Roblin and A. Sibille, "Modeling of the influence of bodyworn antennas upon the path loss variability in UWB WBAN scenarios," in Proceedings of the 30th URSI General Assembly and Scientific Symposium (URSIGASS '11), Istanbul, Turkey, August 2011.

[18] C. E. Baum, "General properties of antennas," Sensor and Simulation Notes 330, 1991.

[19] T. Alves, R. Augustine, P. Quéffélec, M. Grzeskowiak, B. Poussot, and J. M. Laheurte, "Polymeric ferrite-loaded antennas for on-body communications," Microwave and Optical Technology Letters, vol. 51, no. 11, pp. 2530-2533, 2009.

[20] K. Y. Yazdandoost and R. Kohno, "Wireless communications for body implanted medical device," in Proceedings of the AsiaPacific Microwave Conference (APMC '07), December 2007.

[21] S. W. Qu, J. L. Li, Q. Xue, and C. H. Chan, "Wideband cavity-backed bowtie antenna with pattern improvement," IEEE Transactions on Antennas and Propagation, vol. 56, no. 12, pp. 3850-3854, 2008.

[22] T. Alves, R. Augustine, M. Grzeskowiak et al., "BAN antenna design using ferrite polymer composite," in Proceedings of the 3rd European Conference on Antennas and Propagation (EuCAP '09), pp. 965-968, Berlin, Germany, March 2009. 
[23] M. Klemm, I. Z. Kovcs, G. F. Pedersen, and G. Tröster, "Novel small-size directional antenna for UWB WBAN/WPAN applications," IEEE Transactions on Antennas and Propagation, vol. 53, no. 12, pp. 3884-3896, 2005.

[24] W. S. T. Rowe and R. B. Waterhouse, "Reduction of backward radiation for CPW fed aperture stacked patch antennas on small ground planes," IEEE Transactions on Antennas and Propagation, vol. 51, no. 6, pp. 1411-1413, 2003.

[25] H. Kanaya, T. Hashiguchi, R. K. Pokharel, and K. Yoshida, "Sutdy of a CPW-fed slot dipole one-sided directional antenna for UWB systems," in Proceedings of the Asia-Pacific Microwave Conference (APMC '07), December 2007.

[26] Q. Lu, L. Zhou, C. Tan, and G. Lu, "A novel wide beam uwb antenna design for through-the-wall radar," in Proceedings of the International Conference on Microwave and Millimeter Wave Technology (ICMMT'10), pp. 1912-1915, May 2010.

[27] S. Sierra-Garcia and J. J. Laurin, "Study of a CPW inductively coupled slot antenna," IEEE Transactions on Antennas and Propagation, vol. 47, no. 1, pp. 58-64, 1999.

[28] S. Gabriel, R. W. Lau, and C. Gabriel, "The dielectric properties of biological tissues: II. Measurements in the frequency range $10 \mathrm{~Hz}$ to $20 \mathrm{GHz}$," Physics in Medicine and Biology, vol. 41, no. 11, pp. 2251-2269, 1996.

[29] C. Gabriel, "Compilation of dielectric properties of body tissues at RF and microwave frequencies," Tech. Rep. AL/OETR-1996-0037, 1996.

[30] M. Klemm and G. Troester, "EM energy absorption in the human body tissues due to UWB antennas," Progress in Electromagnetics Research, vol. 62, pp. 261-280, 2006. 

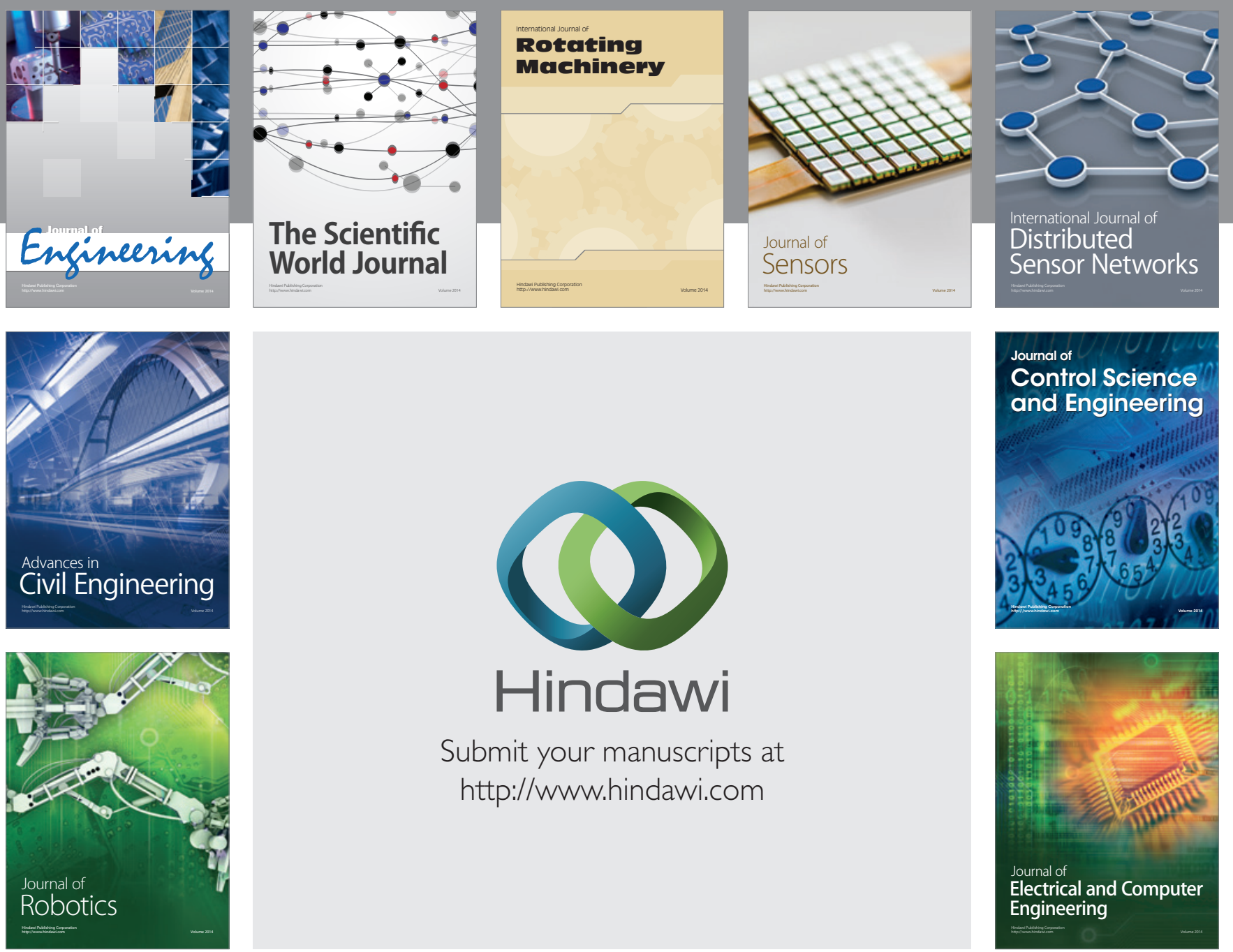

Submit your manuscripts at

http://www.hindawi.com
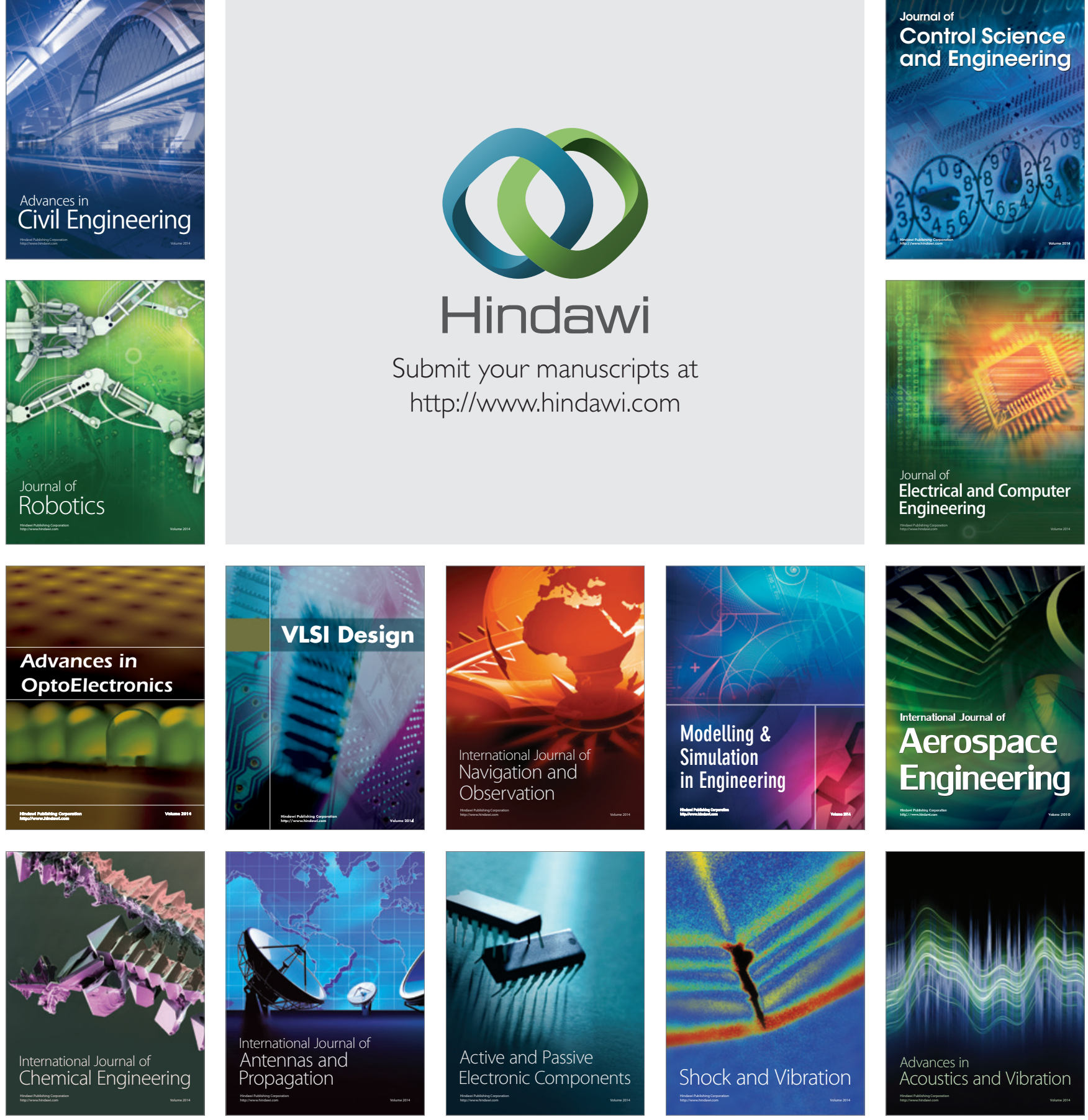